Marcel Bachmann, Vjaceslav Avilov, Andrey Gumenyuk, Michael Rethmeier

\title{
Numerical assessment and experimental verification of the influence of the Hartmann effect in laser beam welding processes by steady magnetic fields
}

Journal article | Accepted manuscript (Postprint)

This version is available at https://doi.org/10.14279/depositonce-10572

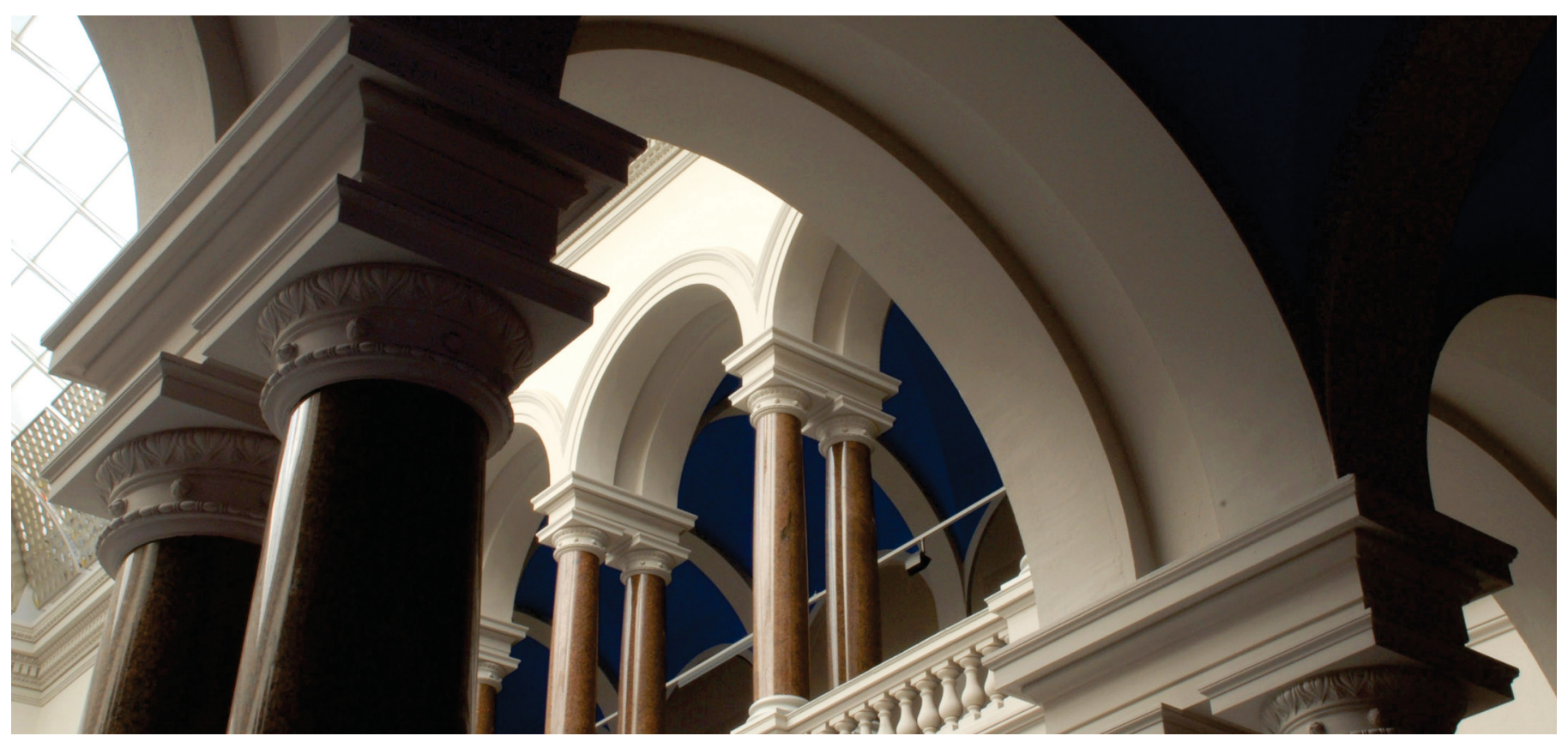

Bachmann, M., Avilov, V., Gumenyuk, A., \& Rethmeier, M. (2016). Numerical assessment and experimental verification of the influence of the Hartmann effect in laser beam welding processes by steady magnetic fields. International Journal of Thermal Sciences, 101, 24-34. https://doi.org/10.1016/j. ijthermalsci.2015.10.030 


\title{
Numerical assessment and experimental verification of the influence of the Hartmann effect in laser beam welding processes by steady magnetic fields
}

\author{
Marcel Bachmann ${ }^{\mathrm{a}, *}$, Vjaceslav Avilov $^{\mathrm{b}}$, Andrey Gumenyuk $^{\mathrm{a}}$, Michael Rethmeier ${ }^{\mathrm{a}, \mathrm{b}}$ \\ ${ }^{a}$ BAM Federal Institute for Materials Research and Testing, \\ Unter den Eichen 87, 12205 Berlin, Germany \\ ${ }^{b}$ Technical University Berlin, Institute of Machine Tools and Factory Management, \\ Pascalstraße 8-9, 10587 Berlin, Germany
}

\begin{abstract}
Controlling the dynamics in the weld pool is a highly demanding challenge in deep-penetration laser beam welding with modern high power laser systems in the multi kilowatt range. An approach to insert braking forces in the melt which is successfully used in large-scaled industrial applications like casting is the so-called Hartmann effect due to externally applied magnetic fields. Therefore, this study deals with its adaptation to a laser beam welding process of much smaller geometric and time scale. In this paper, the contactless mitigation of fluid dynamic processes in the melt by steady magnetic fields was investigated by numerical simulation for partial penetration welding of aluminium. Three-dimensional heat transfer, fluid dynamics including phase transition and electromagnetic field partial differential equations were solved based on temperature-dependent material properties up to evaporation temperature for two different penetration depths of the laser beam. The Marangoni convection in the surface region of the weld pool and the natural convection due to the gravitational forces were identified as main driving forces in the weld pool. Furthermore, the latent heat of solid-liquid phase transition was taken into account and the solidification was modelled by the Carman-Kozeny equation for porous medium morphology.

The results show that a characteristic change of the flow pattern in the melt can be achieved by the applied steady magnetic fields depending on the ratio of magnetic induced and viscous drag. Consequently, the weld bead geometry was significantly influenced by the developing Lorentz forces. Welding experiments with a $16 \mathrm{~kW}$ disc laser with an applied magnetic flux density of around $500 \mathrm{mT}$ support the numerical results by showing a dissipating effect on the weld pool dynamics.
\end{abstract}

Keywords:

electromagnetic weld pool control, Hartmann effect, laser beam welding, Lorentz force, Marangoni flow, natural convection, aluminium

\section{Introduction}

The availability of modern high power laser systems paved the way for a variety of different welding applications into the industrial praxis, e.g. in the automotive and naval industry as well as for large-scaled constructions, power plants, reactor vessels or pipelines. In former times, thick section weld-

*author to whom any correspondence should be addressed

Email address: Marcel.Bachmann@BAM.de (Marcel Bachmann)

Preprint submitted to International Journal of Thermal Sciences ing of metal parts was a classical application for the electron beam (EB) welding technology [1]. Electron beam sources of more than $100 \mathrm{~kW}$ power were developed and used in the industrial production chain. The improvement of the laser beam (LB) sources over the years made the laser beam welding to an attractive alternative due to its efficiency, flexibility and the possibility to weld under atmospheric conditions.

Due to the development of the metal vapour plasma, the classical $\mathrm{CO}_{2}$ laser with a wavelength of $10.6 \mu \mathrm{m}$ is applicable for 
welded layers of up to $20 \mathrm{~mm}$ thickness [2]. A further increase of laser power does not lead to an increase in laser penetration depth. Modern disc and fibre laser deliver output powers of up to $100 \mathrm{~kW}$ (cw) with a wavelength of around $1 \mu \mathrm{m}$ [3]. Those types of lasers allow for the welding of thicknesses of more than $20 \mathrm{~mm}$ per welded layer [4]. A further advantage of the laser beam welding technology in contrast to EB welding is that electromagnetic methods can be exploited to improve the welding process and the weld quality $[4,5]$.

A common issue when joining large structures are the welding distortions depending on the heat input during the processing. When the distortions are larger than the tolerable tolerances, time-consuming and expensive steps of mechanical posttreatment can become necessary. This is especially important for classical arc welding applications but also for multi-layer welding with high accumulated energy input. The bending and angular distortions can be limited by a homogeneous solidification of the weld bead when the side walls of the molten pool are nearly parallel. Thus, the shrinkage associated to the cooling after the welding procedure mainly causes longitudinal and transversal stresses instead.

Characteristically associated to the welding of aluminium alloys is the very high heat conductivity in their liquid state. Thus, the weld beads are very large especially near the surfaces. There, the thermocapillary (Marangoni) convection [7] is the dominating driving mechanism leading to a typical wineglassshape of the weld cross sections. The strong curvature of the weld bead causes inhomogeneous solidification fronts and promotes bending and buckling distortions after cooling down. For the welding with high laser powers, this is especially important in deep penetration welding.

Another issue when welding thick structures is the development of spattering and blow-outs of liquid metal which are caused by very high local melt velocities $[8,9]$. When the surface tension cannot hold the melt, welding defects, e.g. a lack of fusion can occur. This can be avoided by a reduction of the influence of the melt dynamics. Hence, this paper deals with the electromagnetic deceleration of the melt during laser weld-

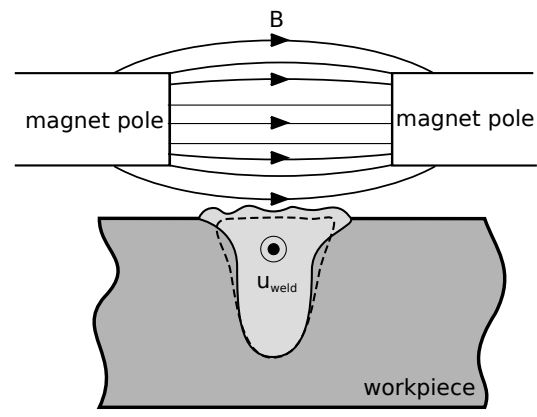

Figure 1: Influence of the applied magnetic field on the weld pool geometry.

ing processes and its influence on the resulting solidification behaviour (see Fig. 1) which is called Hartmann effect.

It is well-known that applied constant or oscillating magnetic fields can effectively decelerate the fluid flow in metal melts [10] and reduce the convective heat transfer consequently $[11,12]$. The movement of an electrically conducting medium perpendicular to an applied magnetic field $\mathbf{B}$ induces an electric current density:

$$
\mathbf{j}_{\mathbf{u}} \sim \sigma \mathbf{u} \times \mathbf{B}
$$

where $\sigma$ and $\mathbf{u}$ are the electric conductivity and the melt velocity, respectively. The interaction of the resulting electric currents with the externally applied magnetic field leads to a Lorentz force distribution being partially directed against the melt velocity. Hence, the corresponding Lorentz force acts like an additional viscous force that dampens the flow velocities. The electromagnetic contribution to the dynamic viscosity of the melt is:

$$
\eta_{\mathrm{EM}}=\sigma B^{2} L^{2}
$$

with the weld bead half width $L$. A measure for the relative importance of magnetically induced drag and viscous forces is the Hartmann number [13]:

$$
\mathrm{Ha}^{2}=\frac{\eta_{\mathrm{EM}}}{\eta}=\frac{\sigma B^{2} L^{2}}{\eta} .
$$

To clarify the relative influence of the induced braking forces on the resulting flow field, their ratio to the flow inertia is decisive. This relation is described by the interaction parameter [13]:

$$
\mathrm{N}=\frac{\mathrm{Ha}^{2}}{\operatorname{Re}}=\frac{\sigma B^{2} L}{\rho U}
$$


$\mathrm{B}=0.25 \mathrm{~T}$

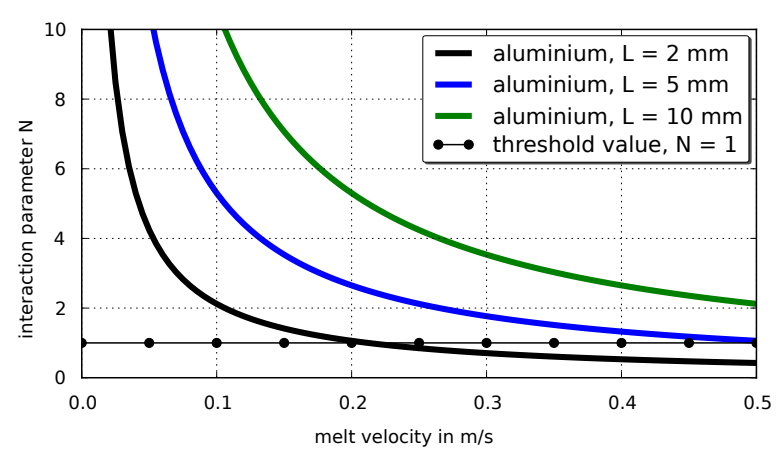

Figure 2: Influence of an applied steady magnetic field of $0.25 \mathrm{~T}$ on the flow field.

with $\operatorname{Re}$ being the Reynolds number and $U$ the velocity magnitude. An estimation of the interaction parameter can be seen in Fig. 2.

It shows, that the effect of an applied magnetic field is larger for wider weld beads as well as for smaller melt velocities, where the flow inertia is lower.

In many industrial processes like continuous casting or crystal growth, steady, travelling and oscillatory magnetic fields are widely used to achieve widespread goals, e.g. a grain refinement, surface stabilization or a flow deceleration in electrically conducting liquids. Reviews on the use of magnetohydrodynamics in materials processing are given in $[14,15]$. Especially the Hartmann effect was also used in crystal growth [16], surface alloying [17] and continuous casting [18] reporting a distinct flow deceleration and a reduction of turbulence levels. Welding-related examples of the application of Lorentz forces in the weld bead are given in [19].

First experimental results of an investigation of the Hartmann effect due to stationary magnetic fields in $\mathrm{CO}_{2}$ laser beam welding were presented in [20]. The half width of the weld bead was around $1 \mathrm{~mm}$ and the magnetic flux density was $40 \mathrm{mT}$ leading to Hartmann numbers $\mathrm{Ha}^{2}$ around 100. A smoothing of the weld seam and humping prevention was observed in dependence of the polarity of the applied DC fields, which was related to thermoelectric currents between material of different temperatures. These currents were independent of the direction of the applied magnetic fields. In a later publication [21], the observed phenomena were explained by an interaction of the $\mathrm{CO}_{2}$ laser plasma with the welded specimen and related to the wavelength of the laser radiation. A more recent work reports on thermoelectric currents during laser beam welding including sheath effects as well as a potential drop within the laserinduced plasma [22]. In conclusion, the scientific importance of the investigation presented in this paper is based on the isolated evaluation of the Hartmann effect. Hence, a disc laser was used for the experimental part in this paper with a ten times shorter wavelength compared to the used $\mathrm{CO}_{2}$ laser in [20].

\section{Setup}

In this investigation, two configurations were investigated numerically, see Figure 3. The first refers to the laboratory scale with optimal conditions for the Hartmann effect, namely a wide weld bead as well as the magnetic field being concentrated in the weld pool (Fig. 3(a)). Hereafter, this setup is referred to as configuration A. The second configuration is devoted to investigate the influence of the Hartmann effect in a more common welding application: the weld seam is smaller and the magnet poles of the DC electromagnet are mounted above the workpiece (Fig. 3(b)). This setup is referred to as configuration B.

The welding experiments were conducted in the configuration seen in Fig. 3(a).

\subsection{Numerical setup}

The numerical simulations were conducted with the commercial finite element solver COMSOL Multiphysics. As the involved physics affect each other and additionally are affected by the temperature-depending material properties (see Fig. 4), a segregated solver was used to solve the energy equation, Navier-Stokes equations with a standard $k-\varepsilon$ turbulence model, and Maxwell equations iteratively. The setup shown in Fig. 3(a) was calculated using a mesh with around 900000 elements, whereas the setup in Fig. 3(b) needed 265000 mesh elements. Using linear elements types for the temperature as well as the 


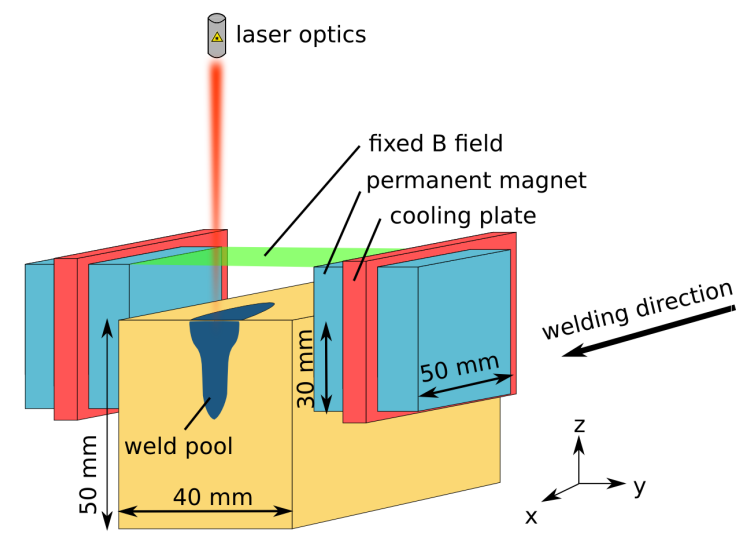

(a)

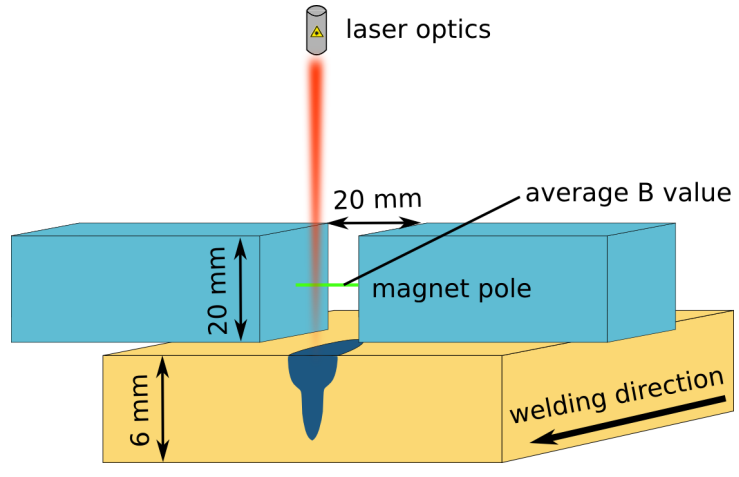

(b)

Figure 3: Setup of the investigated configuration. (a) Permanent magnets mounted on the workpiece. (b) DC electromagnet above the welded specimen.

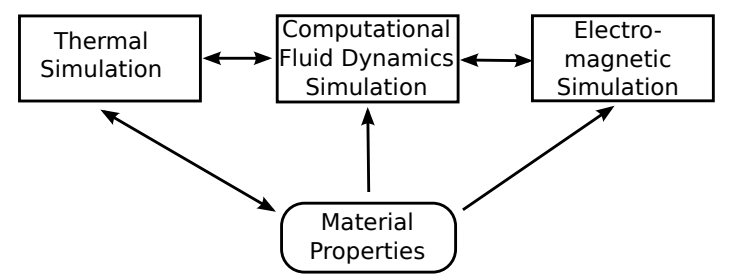

Figure 4: Numerical coupling of heat transport, fluid dynamics and electrodynamics in a welding process simulation.

fluid flow and quadratic shape functions for the electromagnetic part, the total amount of degrees of freedom was around 8100000 and 600000 , respectively. The results were checked for insensitivity of the used meshs and the required relative tolerance in each of the segregated substeps to achieve convergence was 0.001 .

The setup details for the two cases investigated (Fig. 3) are summarized in Tab. 1.

The assumptions for the calculations, the material model as

Table 1: Details for the used setup in the simulations.

\begin{tabular}{rcc}
\hline configuration & A - Fig.3(a) & B - Fig.3(b) \\
material & \multicolumn{2}{c}{ aluminium } \\
material thickness in mm & 50 & 6 \\
\hline welding velocity in $\mathrm{m} / \mathrm{min}$ & 0.5 & 2 \\
penetration depth keyhole in $\mathrm{mm}$ & 21 & 4 \\
magnet pole cross section & $50 \mathrm{~mm} \times 50 \mathrm{~mm}$ & $20 \mathrm{~mm} \times 20 \mathrm{~mm}$ \\
pole distance & $40 \mathrm{~mm}$ & $20 \mathrm{~mm}$ \\
distance pole workpiece & $30 \mathrm{~mm}$ overlap & $2 \mathrm{~mm}$ \\
\hline \hline
\end{tabular}

well as the governing equations with the corresponding boundary conditions are justified in detail in [12]. Therefore, they are listed here only briefly:

- Mass conservation

$$
\nabla \cdot(\rho \mathbf{u})=0
$$

with the mass density $\rho$ and the fluid velocity $\mathbf{u}=(u, v, w)$.

- Momentum conservation

$$
\begin{aligned}
\rho(\mathbf{u} \cdot \nabla) \mathbf{u}= & \nabla \cdot\left[-p \mathbf{I}+\left(\eta+\eta_{T}\right)\left(\nabla \mathbf{u}+(\nabla \mathbf{u})^{T}\right)\right. \\
& \left.-\frac{2}{3}\left(\eta+\eta_{T}\right)(\nabla \cdot \mathbf{u}) \mathbf{I}-\frac{2}{3} \rho k \mathbf{I}\right]+\mathbf{F}
\end{aligned}
$$

with the pressure $p$, turbulent viscosity $\eta_{T}$ and the source term $\mathbf{F}$.

$$
\begin{aligned}
\mathbf{F}= & \rho \mathbf{g} \beta\left(T-T_{\text {melt }}\right)-c_{1} \frac{\left(1-f_{\mathrm{L}}\right)^{2}}{f_{\mathrm{L}}^{3}+c_{2}}\left(\mathbf{u}-\mathbf{u}_{\text {weld }}\right) \\
& +\mathbf{j} \times \mathbf{B}
\end{aligned}
$$

with the first term on the right-hand side (RHS) being the buoyancy term with the gravitational constant $\mathbf{g}$ and the coefficient of thermal expansion $\beta$. The second term on the RHS decelerates the flow in the solid region to the welding velocity (Carman-Kozeny equation [23]). There, $f_{L}$ is the liquid fraction and $c_{1}$ and $c_{2}$ are chosen constants. The third term on the RHS refers to the Lorentz force with the electric current density $\mathbf{j}$ and the magnetic flux density $\mathbf{B}$. 
Table 2: Material properties of pure aluminium at melting temperature $T_{\text {melt }}$ $[24,25]$.

\begin{tabular}{rccc}
\hline \hline Material property & & Value & Unit \\
\hline Melting temperature & $T_{\text {melt }}$ & 933 & $\mathrm{~K}$ \\
Evaporation temperature & $T_{\text {evap }}$ & 2700 & $\mathrm{~K}$ \\
\hline Mass density & $\rho$ & 2380 & $\mathrm{~kg} \mathrm{~m}^{-3}$ \\
Heat capacity & $C_{p}$ & 1180 & $\mathrm{~J} \mathrm{~kg}^{-1} \mathrm{~K}^{-1}$ \\
Latent heat of fusion & $H_{\mathrm{f}}$ & $3.97 \times 10^{5}$ & $\mathrm{~J} \mathrm{~kg}^{-1}$ \\
Heat conductivity & $\lambda$ & 91 & $\mathrm{~W} \mathrm{~m}^{-1} \mathrm{~K}^{-1}$ \\
Dynamic viscosity & $\eta$ & $1.1 \times 10^{-3}$ & $\mathrm{~Pa} \mathrm{~s}^{-1}$ \\
Surface tension & $\gamma$ & 0.871 & $\mathrm{~N} \mathrm{~m}^{-1}$ \\
Marangoni coefficient & $\partial \gamma / \partial T$ & $-1.55 \times 10^{-4}$ & $\mathrm{~N} \mathrm{~m}^{-1} \mathrm{~K}^{-1}$ \\
Electrical resistivity & $\rho_{e l}=\sigma^{-1}$ & $24.77 \times 10^{-8}$ & $\Omega \mathrm{m}$ \\
\hline \hline
\end{tabular}

Further equations of the standard $k-\varepsilon$ turbulence model are solved for the calculation of the turbulent viscosity

$$
\eta_{T} \propto \frac{k^{2}}{\varepsilon}
$$

with the turbulent kinetic energy $k$ and the turbulent dissipation rate $\varepsilon$.

- Energy conservation

$$
\rho C_{p} \mathbf{u} \cdot \nabla T=\nabla \cdot(\lambda \nabla T)
$$

Here, $C_{p}, T$, and $\lambda$ are heat capacity, temperature and heat conductivity. An effective formulation for the heat capacity was used to account for the latent heat of melting and solidification.

- Electromagnetic equations

$$
\begin{aligned}
\nabla \times \mathbf{B} & =\mu_{0} \mathbf{j}, \\
\nabla \times \mathbf{E} & =0 .
\end{aligned}
$$

with the magnetic permeability in vacuum $\mu_{0}$ and the electric field $\mathbf{E}$. The movement of conducting particles in the magnetic field induces a current density according to Ohm's law without further external sources.

$$
\mathbf{j}=\sigma(\mathbf{E}+\mathbf{u} \times \mathbf{B})
$$

The boundary conditions are explained in detail in [12]. The most important assumptions are listed below:
- Half-symmetry was exploited.

- Temperature-dependent material model (see Tab. 2). As temperature-dependent material properties for $\mathrm{AlMg} 3$, especially in the liquid state are hardly documented in the literature, the values for available pure aluminium were chosen. For low-alloyed Al, the thermophysical properties are comparable in the liquid state. The values of the solid material may differ slightly, but as the processes happening inside the weld pool are of major interest, this was neglected. Even larger differences occur for the solid state electric conductivity (a factor of around 2), but Ohmic losses in the specimen were not regarded in this study.

- Fixed geometry and keyhole surfaces. Hence, it is assumed that the welding conditions as well as the dynamics inside the molten pool allow for a stable process.

- Keyhole temperature set to evaporation temperature.

- No flow normal to the keyhole wall.

- Non-viscous flow along the keyhole wall and surface.

- Marangoni flow at the upper surface.

$$
\begin{aligned}
\eta \frac{\partial u}{\partial z} & =\frac{\partial \gamma}{\partial T} \frac{\partial T}{\partial x} \\
\eta \frac{\partial v}{\partial z} & =\frac{\partial \gamma}{\partial T} \frac{\partial T}{\partial y}
\end{aligned}
$$

with the surface tension $\gamma$.

- The magnetic flux densities referred to in the results section are the constant values at the magnet edge (configuration $\mathrm{A}$ ) and the average value in the magnet pole centreline (configuration B), see also Fig. 3.

The chosen boundary conditions at the keyhole walls imply, that the recoil pressure was ideally balanced by surface tension forces. As the article mainly focuses on the interaction of electromagnetic fields with fluid dynamic effects, an elementary model of the laser beam welding process was implemented. In this configuration, the keyhole is subjected to free slip conditions and its geometry is much smaller than the weld 
pool. Therefore, the influence of the keyhole shape on the flow field is negligible. Nevertheless, the heat input and the resulting weld pool dimensions depend on the keyhole radii, which are a kind of free model parameter to fit the experimental observations in terms of the weld pool dimensions by applying evaporation temperature at the keyhole wall.

\subsection{Experimental setup}

The experiments were conducted in the setup seen in Fig. 3(a). Two NdFeB permanent magnets were mounted on both sides of the workpiece with a water-cooled ferritic plate in between to avoid overheating and subsequent demagnetization. The most important welding parameters for the experiments are summarized in Tab. 3.

Table 3: Details for the used setup in the welding experiments.

\begin{tabular}{rc}
\hline \hline geometric setup & as shown in Fig. 3(a) \\
\hline material & AlMg3 \\
laser source & Trumpf TruDisk 16002 \\
wavelength & $1030 \mathrm{~nm}$ \\
fibre diameter & $200 \mu \mathrm{m}$ \\
focus length & $300 \mathrm{~mm}$ \\
laser incident angle & $18^{\circ}$ \\
laser power & $16 \mathrm{~kW}$ \\
focus position & $-4 \mathrm{~mm}$ \\
welding velocity & $0.5 \mathrm{~m} / \mathrm{min}-1 \mathrm{~m} / \mathrm{min}$ \\
shielding gas & $\mathrm{Ar}$ \\
focus diameter & $300 \mu \mathrm{m}$ \\
magnetic flux density & $500 \mathrm{mT}$ \\
\hline \hline
\end{tabular}

\section{Results}

\subsection{Numerical results}

\subsubsection{Electric current density distribution}

The electric current density distribution in the workpiece and the weld pool depends on the local quantities of the electric conductivity, the flow velocity and the strength of the magnetic field. Outside the weld pool, the electric current density in the simulations is a consequence of the constant velocity difference between the workpiece and the magnets, which is the welding speed, see Fig. 5(a). This is due to the Euler approach,
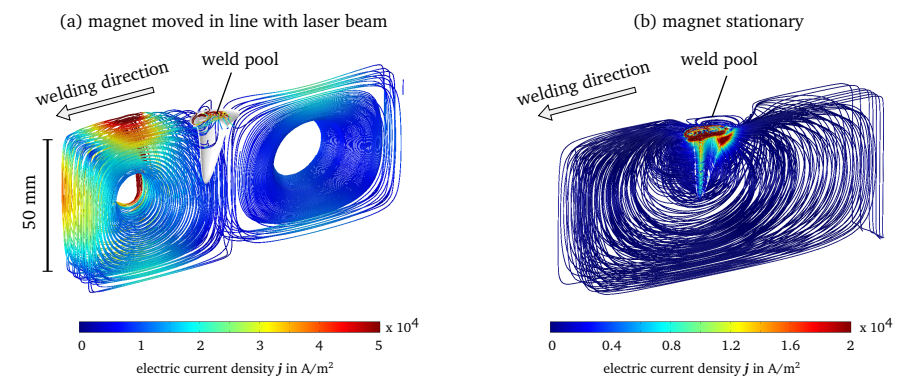

Figure 5: Exemplary electric current density in the workpiece due to the presence of a moving electrically conducting medium and a transverse stationary magnetic field for $\mathrm{B}=0.5 \mathrm{~T}$.
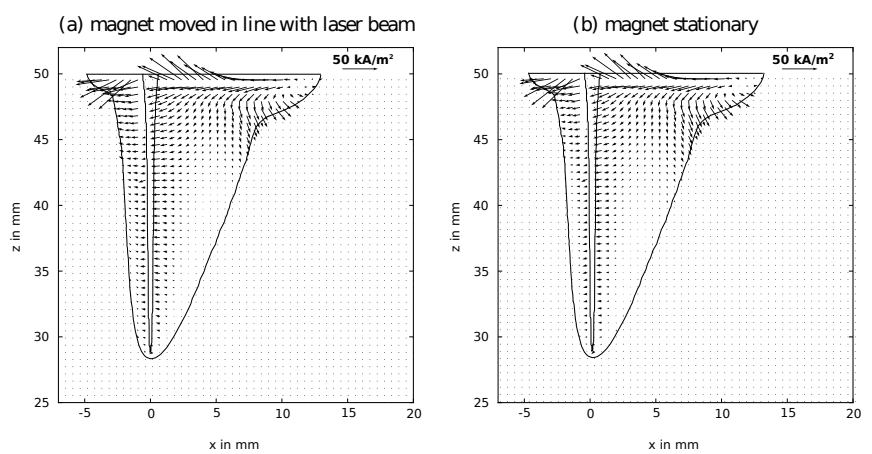

Figure 6: Exemplary electric current density in the weld bead due to the presence of a moving electrically conducting medium and a transverse stationary magnetic field for $\mathrm{B}=0.5 \mathrm{~T}$.

which is usual in computational fluid dynamics. In the experiments, the used permanent magnets were mounted stationary at the sides of the workpiece. Consequently, the velocity difference between the magnets and the solid material are vanishing, which causes a qualitative change of the behaviour of the electric current densities in the whole workpiece. The differences in the electric current density distributions between both cases with the magnets moved in line with the laser beam and the magnets being mounted stationary at the workpiece are shown in Fig. 5.

Nevertheless, Fig. 6 shows, that the electric current density remains nearly the same inside the weld pool. The reason is, that the flow velocities, which are essential for the development of the electric currents, are up to two orders of magnitude larger than the welding speed. Therefore, the differences of the resulting Lorentz forces are negligible and the results of the computer simulations with magnets moving in line with the laser 

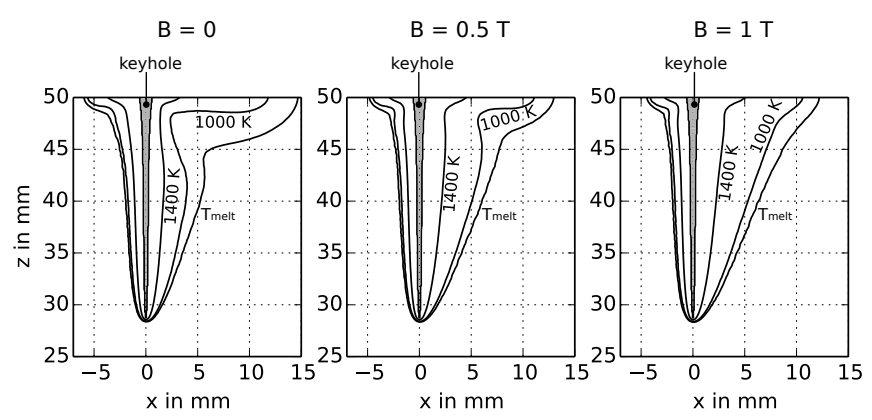

Figure 7: Isotherms in the symmetry plane of the weld pool for three cases of partial penetration welding of aluminium with a laser penetration depth of around $21 \mathrm{~mm}$ : magnet off, $\mathrm{B}=0.5 \mathrm{~T}$, and $\mathrm{B}=1.0 \mathrm{~T}$.

are comparable to the conducted experiments with locally fixated permanent magnets.

\subsubsection{Configuration A}

Selected temperature isolines as well as the flow streamlines of the simulations of partial penetration welding of aluminium without and with applied magnetic flux densities of $0.5 \mathrm{~T}$ and $1 \mathrm{~T}$ are shown in Fig. 7 and Fig. 8. The Marangoni flow causes a flow in the surface-near region, that is directed radially outward from the keyhole to the outer weld pool boundary. Below the surface, the flow direction is opposite due to the law of mass continuity. Thus, the flow reversal affects the geometry of the weld bead and gives it a wineglass-like shape in both the longitudinal as well as the transversal cross sections with a strong curvature of the solidification isotherm especially in the weld pool rear. This behaviour was observed in different welding simulations and for different materials [26, 27]. The natural convection supports that effect by an upwelling flow near the keyhole. The lower part of the weld pool is mainly characterized by a horizontal flow around the keyhole.

Applying a steady magnetic field transverse to the welding direction mitigates the influence of the Marangoni flow on the weld pool dynamics. The flow velocities in the upper part of the weld pool are smaller and the region of the Marangoni vortex is moved towards the weld pool surface. The magnetic braking due to the Hartmann effect evokes a more two-dimensional flow field in wide parts of the weld pool, see Fig. 9 which in the end produces a less curved solidification isotherm. Significant

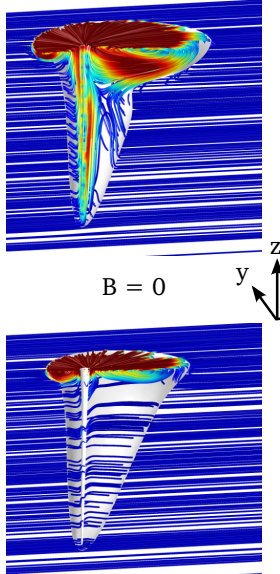

$\mathrm{B}=1.0 \mathrm{~T}$

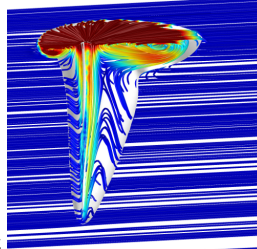

$\mathrm{B}=0.2 \mathrm{~T}$

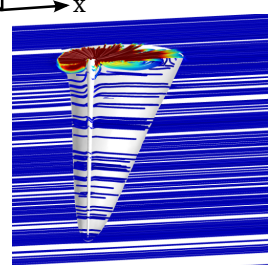

$\mathrm{B}=2.0 \mathrm{~T}$

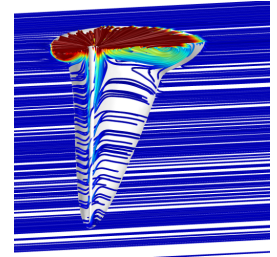

$\mathrm{B}=0.5 \mathrm{~T}$

welding direction $\longrightarrow$

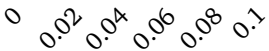
velocity magnitude in $\mathrm{m} / \mathrm{s}$
Figure 8: Flow streamlines coloured with the velocity magnitude. Velocity values above $0.1 \mathrm{~m} / \mathrm{s}$ are coloured in red.

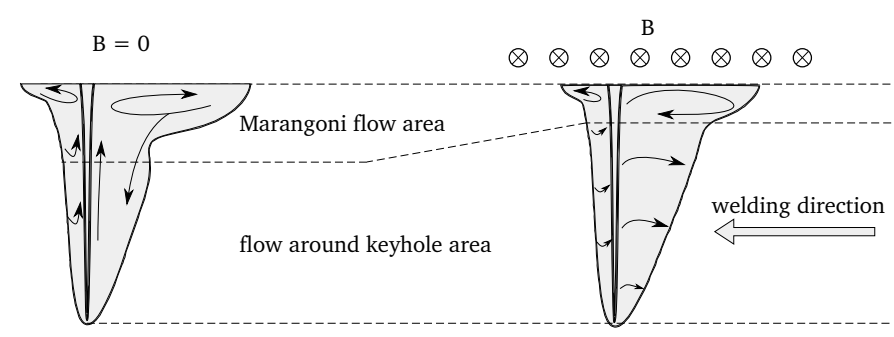

Figure 9: Flow scheme in the weld pool without (left) and with applied magnetic field (right).

changes in the flow field are visible already for magnetic flux densities around $0.5 \mathrm{~T}$. A further increase of the magnetic flux density changes only the weld pool dimensions but not the flow characteristics in the weld pool.

The weld pool width to length ratio for increasing magnetic flux densities is shown in Fig. 10. A clear tendency to shorter and narrower weld pool dimensions was observed for larger applied magnetic fields which is due to the mitigated dynamics and thus less influence of the convective motions in the melt. The corresponding ratio of weld pool width to length also reduces. The values obtained by a coupled simulation for different magnetic flux densities are compared to a theoretical case, where only convective heat transport is assumed by the welding speed $u_{\text {weld }}$ in the whole domain and local velocity variations are neglected. This can be seen as a state of ideal flow deceleration that will not be reached in real experiments, and its ratio 


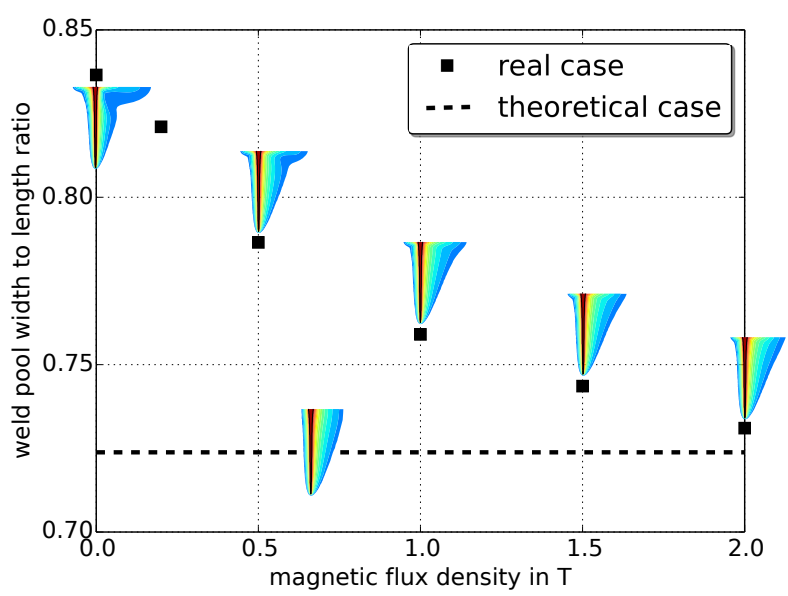

Figure 10: Weld pool width to length ratio for increasing magnetic flux density compared to a theoretical case, assuming only heat conduction with welding speed in the whole calculation domain.

value is the limiting value for the investigated case. The weld pool dimensions for that case were found by the solution of the heat conduction equation with constant convective term.

The braking of the flow velocities is also associated to a decrease in the turbulence levels reached and thus also in the turbulent viscosity, see Fig.11. In the reference case without applied magnetic fields, the turbulent viscosity is especially high in the regions of the recirculation of the flow in the upper part of the weld pool. For a magnetic flux density of $0.5 \mathrm{~T}$, its values have dropped significantly, although the highest values in this case are still observable being considerable above the values of the molecular viscosity.

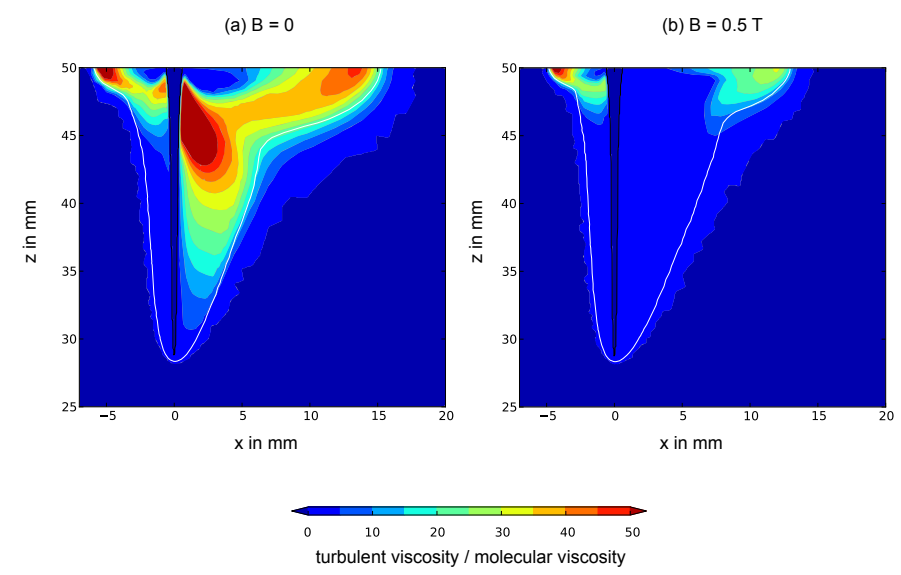

Figure 11: Ratio of the turbulent viscosity and the molecular viscosity in the weld pool for the reference case $(\mathrm{B}=0)$ and for $\mathrm{B}=0.5 \mathrm{~T}$.

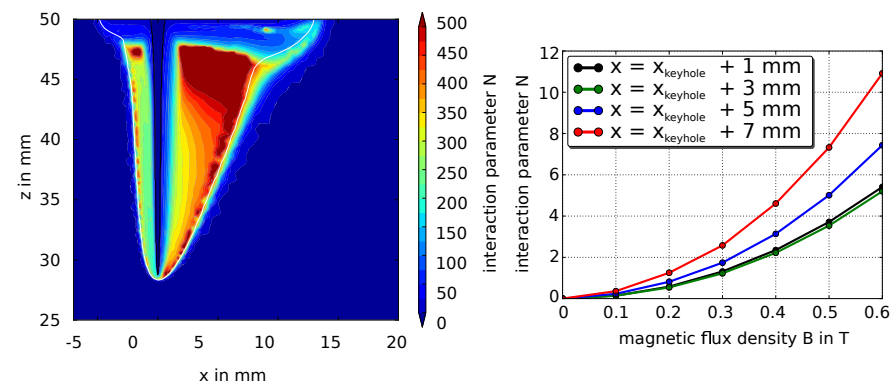

Figure 12: Left: Interaction parameter in the weld symmetry section for a magnetic flux density of $0.5 \mathrm{~T}$ based on an approximate weld pool half width of $8 \mathrm{~mm}$. Right: Distribution of the interaction parameter at the weld surface for increasing magnetic flux density.

The interaction parameter for the case $\mathrm{B}=0.5 \mathrm{~T}$ and its distribution at discrete locations on the surface behind the keyhole for increasing magnetic flux densities are shown in Fig. 12. Due to the high velocities near the surface, the values of $\mathrm{N}$ are much smaller there compared to the area in the depth of the weld pool. Along the weld surface, the interaction number is single-digit and even below unity in some places. The highest flow velocities occur around $3 \mathrm{~mm}$ behind the keyhole, where the interaction number is smallest. Correspondingly, the influence of the induced braking forces is minor there. Nevertheless, the influence of the induced Lorentz forces is large enough to have enough impact on the global flow field to have an effect on the weld pool geometry.

\subsubsection{Configuration $B$}

The comparison between configurations A and B in Fig. 13 in terms of the normalized magnetic flux density shows, that the magnetic field in the weld pool in configuration B is smaller
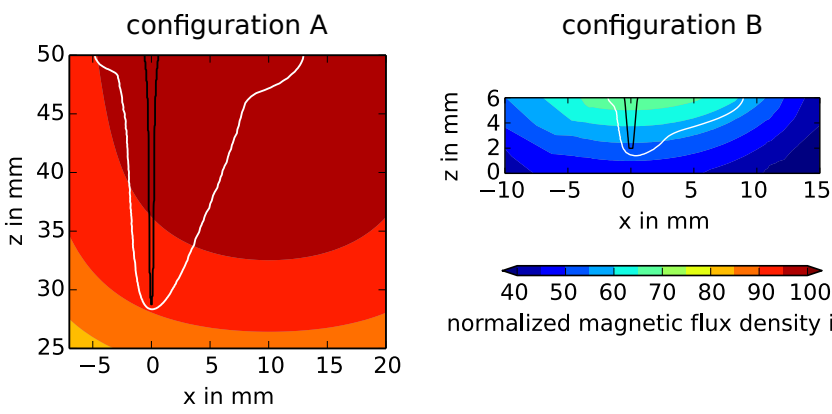
normalized magnetic flux density in \%

Figure 13: Normalized magnetic flux densities for configuration A and B in \% of the applied field strength, see Fig. 3 

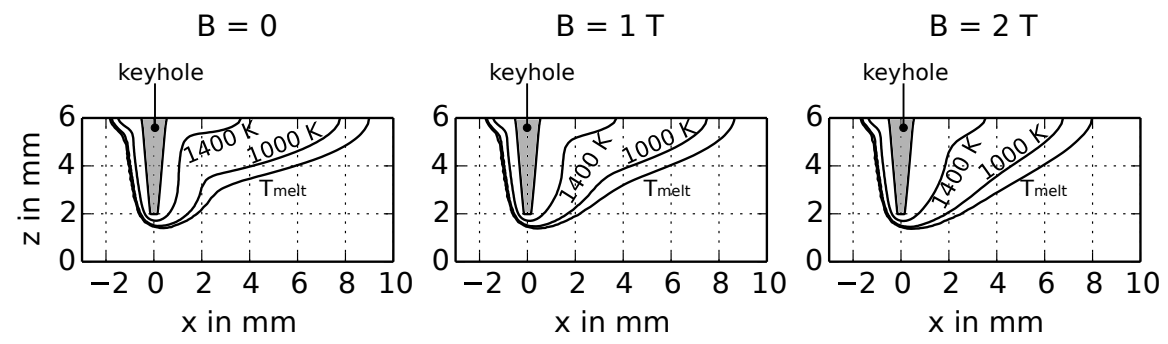

Figure 14: Isotherms in the symmetry plane of the weld pool for three cases of partial penetration welding of aluminium with a laser penetration depth of around $4 \mathrm{~mm}$ : magnet off, $\mathrm{B}=1 \mathrm{~T}$, and $\mathrm{B}=2 \mathrm{~T}$.

than the boundary reference value (around $65 \%$ ) and also less homogeneous in depth compared to configuration A, where the magnetic flux density is nearly constant. One could imagine configuration A being the laboratory case with permanent magnets mounted directly to the welded plates, and configuration $\mathrm{B}$ the one that works with a movable electromagnetic system being mounted above the workpiece.

The simulation results for partial penetration welding in $6 \mathrm{~mm}$ thick aluminium with a keyhole penetration of $4 \mathrm{~mm}$ can be seen in Fig. 14 and Fig. 15. The magnetic flux density values refer to the applied boundary condition value. The results obtained for the temperature as well as for the velocities in the weld pool are qualitatively comparable to that for configuration $\mathrm{A}$. The flow characteristics is still dominated by the strong influence of the Marangoni flow. Increasing the magnetic flux density also leads to a shortening of the weld pool dimensions and a flow that is less disturbed by turbulence and vortices, especially in the lower part of the weld pool where the flow velocities are smaller than near the surface. A comparison of the flow fields in sections 3.1.2 and 3.1.3 shows, that higher values of the magnetic field are needed for smaller weld beads to obtain a similar influence on the velocities and solidification isotherms (cf. eq. (4)). A consequence of the Hartmann effect is the disappearance of the pronounced curvature of the weld pool rear shape as well as a deceleration of the flow velocities in wide areas of the molten zone.

In analogy to configuration $\mathrm{A}$, the simulation results in the symmetry plane as well as on the surface for the interaction parameter $\mathrm{N}$ describing the quantitative influence of the braking

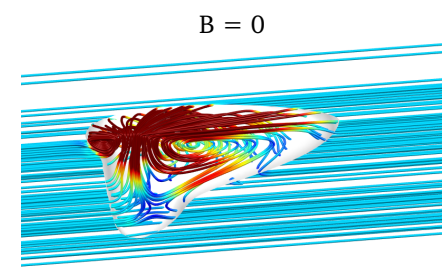

$\mathrm{B}=1.0 \mathrm{~T}$
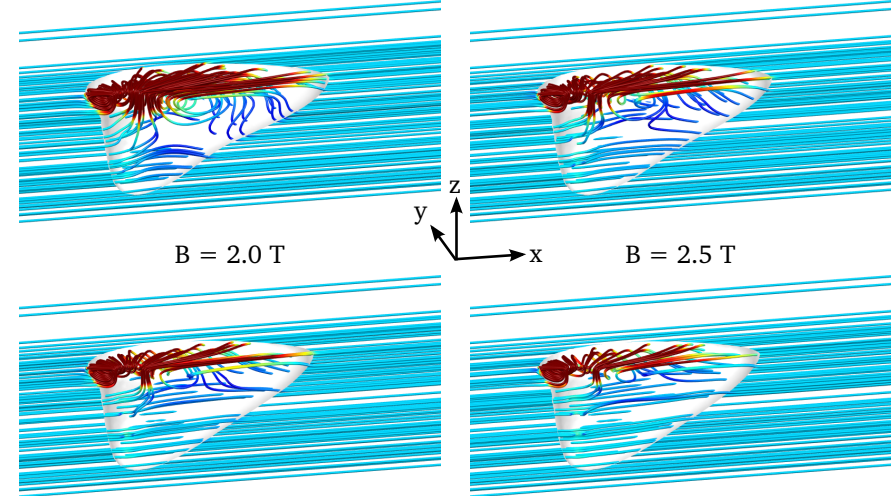

$\mathrm{B}=3.0 \mathrm{~T}$
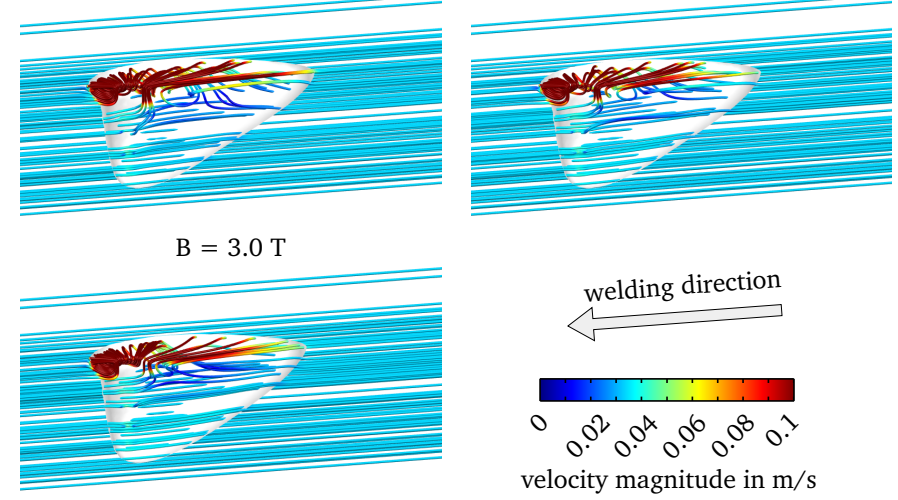

Figure 15: Flow streamlines coloured with the velocity magnitude. Velocity values above $0.1 \mathrm{~m} / \mathrm{s}$ are coloured in red.
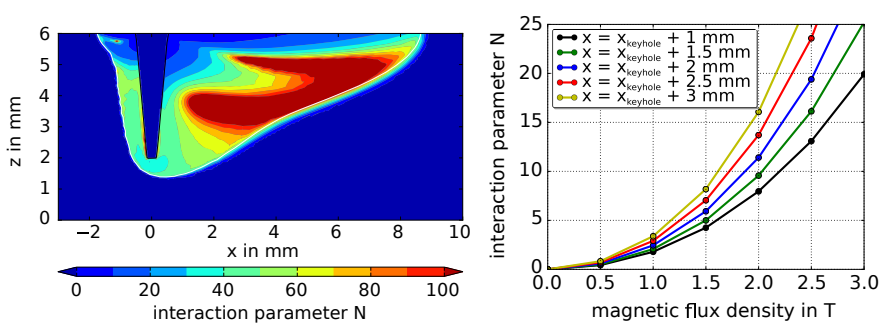

Figure 16: Left: Interaction parameter in the weld symmetry section for a magnetic flux density of $1.0 \mathrm{~T}$ based on an approximate weld pool half width of $3.2 \mathrm{~mm}$. Right: Distribution of the interaction parameter at the weld surface for increasing magnetic flux density. 
Lorentz forces on the flow field are shown in Fig. 16 for configuration B. Again, the highest values of $\mathrm{N}$ are reached below the circulation zone due to the Marangoni vortex, where the flow velocities are moderate and thus the ratio of electromagnetic braking to viscous effects is high. Decisive for the flow control especially near the surface is the region of the Marangoni vortex, see the right diagram in Fig. 16. It reveals that beginning with a magnetic flux density of approximately $1 \mathrm{~T}$, the interaction parameter rises to values significantly larger than one. Hence, a clear mitigating effect on the flow velocities is expectable, which is backed by the results obtained in Fig. 14.

Compared to configuration $\mathrm{A}$, the weld bead shortening due to the Hartmann effect is smaller for configuration $\mathrm{B}$, which can be explained by the higher welding speed and thus a relatively higher convective heat transport influence in reverse processing direction there.

\subsection{Experimental results}

As the experimental setup was the same in an earlier publication, the distribution of the magnetic flux density in the symmetry plane measured by a Hall sensor before welding shown in Fig. 17 was taken from [12]. It shows, that the maximum value is around $0.5 \mathrm{~T}$. Furthermore, the magnetic flux density can be seen as constant for the weld pool taking into account that the typical weld pool dimensions of the previous sections are small compared to the dimensions of the permanent magnet.

The weld surface as well as macrographs at different positions in front of, inside and behind the mounted permanent

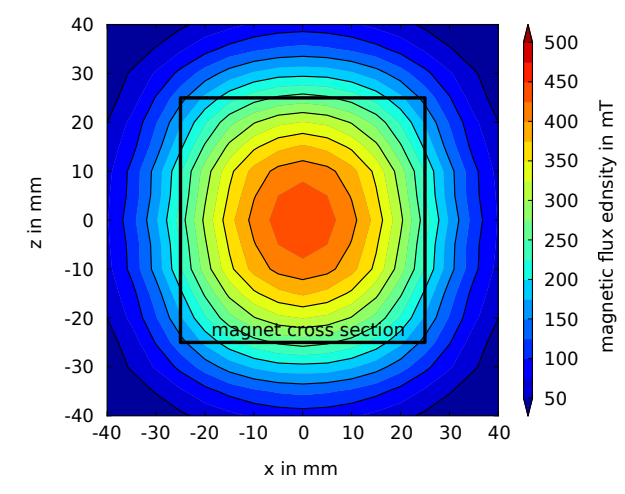

Figure 17: Distribution of the magnetic flux density in the symmetry plane.

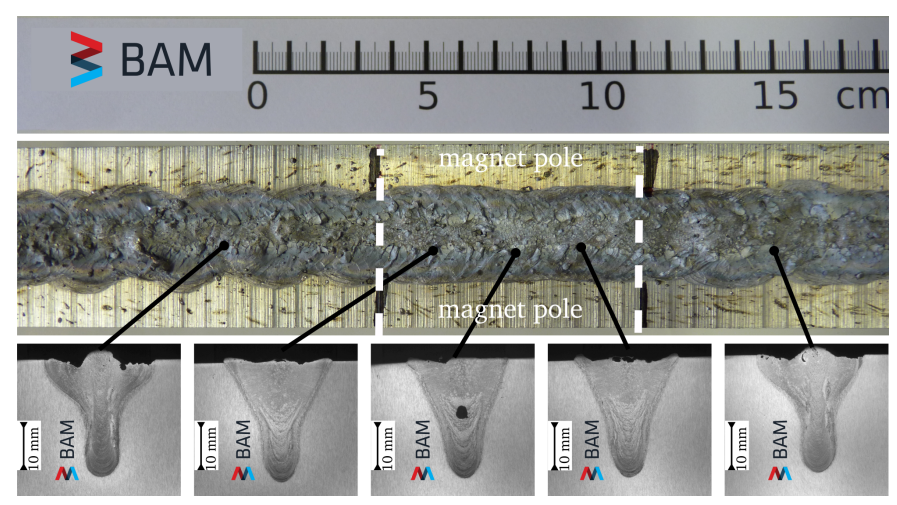

(a) $u_{\text {weld }}=0.5 \mathrm{~m} / \mathrm{min}$

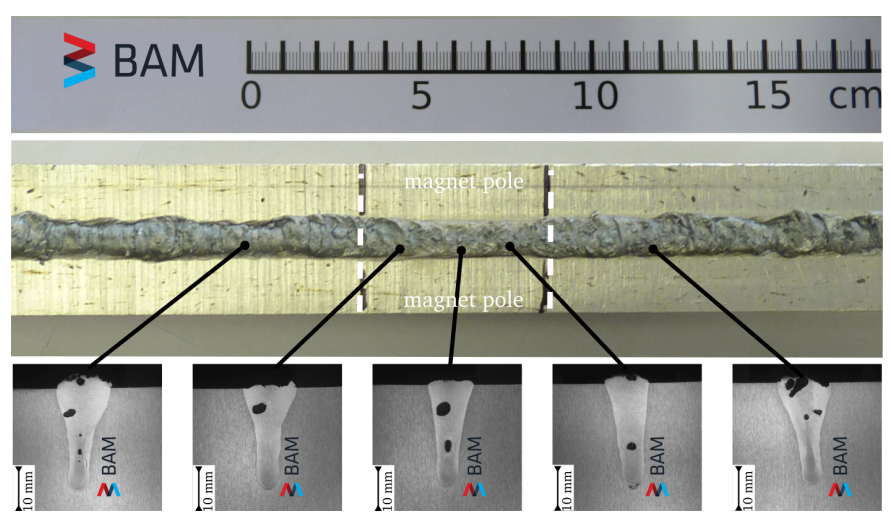

(b) $u_{\text {weld }}=1 \mathrm{~m} / \mathrm{min}$

Figure 18: Experimental results for different welding speeds.

magnets can be seen in Fig. 18 for two different welding speeds made with the aluminium alloy AlMg3. The test parameters are summarized in Tab. 3. In Fig. 18(a), the weld penetration depth is around $25 \mathrm{~mm}$. Strong Marangoni convection at the free surface causes a significant curvature of the solidification isotherm in the macrographs outside the magnetic field. The weld surface is very wavy and irregular due to the dynamics inside the weld pool. Consequently, the weld cross sections show a nailhead geometry in the case without applied magnetic field. The cross sections with magnetic field applied show a $\mathrm{V}$ profile that points to a flow deceleration under the influence of the Lorentz forces and thus lower flow dynamics inside. Additionally, the weld surface quality is smoother compared to the reference case without magnetic field.

The welding tests were repeated with a welding speed of $1 \mathrm{~m} / \mathrm{min}$, see Fig. 18(b). As solidification time becomes smaller with increasing the welding speed, the experimental results in 
Fig. 18(b) suffer to a larger extent from porosity, whose reduction was not the goal of the present investigation. It shows, that the trends to a smoother weld surface and more parallel side walls in the cross sections remain the same under the influence of an applied magnetic field. Nevertheless, the influence of the induced electromagnetic forces is much less pronounced here as the characteristic length of the weld pool is smaller when compared to the lower welding speed. That observation fits very well to the numerical results in sections 3.1.2 and 3.1.3.

A comparison between the numerically obtained weld cross sections (section 3.1.2) and those from welding experiments (18(a)) is shown in Fig. 19. Compared are two cases: the reference case without applied magnetic field and the case corresponding to the experimental magnetic flux density of $500 \mathrm{mT}$. The results are in good accordance in terms of qualitatively reproducing the solidification line as well as the tendency in geometry change between the reference case and the case with applied magnetic field.

Further experiments, with the welding direction turned, showed that the direction of polarisation of the magnetic field is insignificant for the welding results, according to the theory of the Hartmann effect, as long as it is aligned vertical to the welding direction.

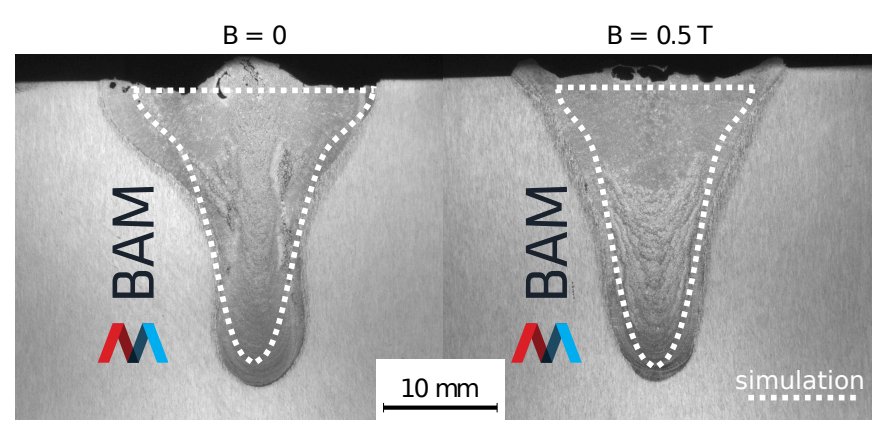

Figure 19: Comparison between experimentally (Fig. 18(a)) and numerically obtained weld cross sections (section 3.1.2).

\section{Summary \& conclusions}

The present investigation deals with the numerical assessment and experimental verification of the interaction of an externally applied steady magnetic field perpendicular to the welding direction with the weld pool dynamics in thick section partial penetration laser beam welding of aluminium with a thin disc laser.

The presented numerical results show a clear influence of the induced electromagnetic forces on the flow velocities and the weld pool shape. The changes in the convection in the melt cause a shortening and also a narrowing of the weld bead. Experimentally, a positive influence of the reduced weld pool dynamics on the weld bead shape and the weld surface was also visible.

It was shown that the investigated effect is primary based on an effective braking of the local flow velocities due to the nature of the generation of the flow-dependent induced electric current density. Hereby associated to the liquid metal flow deceleration is a mitigation of the turbulence levels in the weld pool, which was numerically quantified by the turbulent viscosity values.

According to the definition of the Hartmann number $\mathrm{Ha}^{2}$, it was affirmed, that the Hartmann effect works more efficiently for larger weld bead dimensions, which usually restricted its use to processes of larger scale, e.g. crystal growth and casting applications. Referred to deep penetration welding, an influence of the induced Lorentz forces was remarkable, when the Hartmann number in the area of the Marangoni vortex exceeded the flow inertia associated to interaction numers being larger than unity. Subsequently, common industrial welding applications aside from shipyards or heavy industry with corresponding smaller weld pool dimensions and a magnet that would be mounted above the weld pool would require an equivalently larger magnetic field.

It can be concluded, that the reduced influence of the Marangoni convection on the curvature of the solidified weld bead is favourable in terms of a homogeneous mechanical stress distribution in the weld specimen thus being able to decrease the susceptibility level against welding-induced distortions. 


\section{Acknowledgements}

Financial funding of the Deutsche Forschungsgemeinschaft DFG (Bonn, Germany) under Grant DFG GU 1211/2-1 and the German Allianz Industrie Forschung (AiF), Grant $17.265 \mathrm{~N}$ is gratefully acknowledged.

\section{References}

[1] A. Kohyama, Y. Arata, M. Tomie, N. Igata, Electron beam welding of titanium and Ti-6AL-4V thick plates, J. Nucl. Mater. 122 (1984) 772 776.

[2] E. Beyer, Schweißen mit Laser, Springer, 1995.

[3] C. Thomy, F. Vollertsen, T. Seefeld, Schweißen mit HochleistungsFaserlasern - Was sind die Vorteile, wo sind die Grenzen?, Laser Tech. J. 3 (2005), $28-31$.

[4] V. Avilov, A. Gumenyuk, M. Lammers, M. Rethmeier, PA position full penetration high power laser beam welding of up to $30 \mathrm{~mm}$ thick $\mathrm{AlMg} 3$ plates using electromagnetic weld pool support, Sci. Technol. Weld. Joining 17 (2012), $128-133$.

[5] M. Bachmann, V. Avilov, A. Gumenyuk, M. Rethmeier, Experimental and Numerical Investigation of an Electromagnetic Weld Pool Control for Laser Beam Welding, Phys. Proc. 56 (2014), 515 - 524.

[6] D. Radaj, Eigenspannungen und Verzug beim Schweißen, DVS-Verlag, Düsseldorf, 2002.

[7] E. Magyari, A.J. Chamkha, Exact analytical results for the thermosolutal MHD Marangoni boundary layers, Int. J. Therm. Sci. 47 (2008), 848 857.

[8] Y. Kawahito, M. Mizutani, S. Katayama, High quality welding of stainless steel with $10 \mathrm{~kW}$ high power fibre laser, Sci. Technol. Weld. Joi. 14 (2009), $288-294$.

[9] A.F.H. Kaplan, J. Powell, Spatter in laser welding, J. Laser Appl. 23 (2011), 032005.

[10] V. Bojarevics, J.A. Freibergs, E.I. Shilova, E.V. Shcherbinin, Electrically induced vortical flows, Kluwer Academic Publishers, 1989.

[11] M. Sathiyamoorthy, A. Chamkha, Effect of magnetic field on natural convection flow in a liquid gallium filled square cavity for linearly heated side wall(s), Int. J. Therm. Sci. 49 (2010), 1856 - 1865.

[12] M. Bachmann, V. Avilov, A. Gumenyuk, M. Rethmeier, About the influence of a steady magnetic field on weld pool dynamics in partial penetration high power laser beam welding of thick aluminium parts, Int. J. Heat Mass Tran. 60 (2013), $309-321$.

[13] R. Moreau, Magnetohydrodynamics, Kluwer Academic Publisher, 1990.

[14] P.A. Davidson, Magnetohydrodynamics in materials processing, Annu. Rev. Fluid Mech. 31 (1999), 273 - 300.

[15] S. Asai, Recent development and prospect of electromagnetic processing of materials, Sci. Technol. Adv. Mater. 1 (2000), $191-200$.
[16] B.H. Dennis, G.S. Dulikravich, Magnetic field suppression of melt flow in crystal growth, Int. J. Heat Mass Transfer 23 (2002), 269 - 277.

[17] O. Velde, R. Gritzki, R. Grundmann, Numerical investigation of Lorentz force influenced Marangoni convection relevant to aluminum surface alloying, Int. J. Heat Mass Transfer 44 (2001), 2751 - 2762.

[18] K. Timmel, S. Eckert, G. Gerbeth, Experimental investigation of the flow in a continuous-casting mold under the influence of a transverse, direct current magnetic field, Metal. Mater. Trans. B 42 (2011), 68 - 80.

[19] F. Vollertsen, C. Thomy, Magnetic stirring during laser welding of aluminium, J. Laser Appl. 18 (2006), 28 - 34.

[20] M. Kern, P. Berger, H. Hügel, Magneto-fluid dynamic control of seam quality in $\mathrm{CO}_{2}$ laser beam welding, Weld. J. (2000), 72s - 78s.

[21] G. Ambrosy, V. Avilov, P. Berger, H. Hügel, Laser induced plasma as a source for an intensive current to produce electromagnetic forces in the weld pool, XVI International Symposium on Gas Flow, Chemical Lasers, and High-Power Lasers (2007).

[22] H. Zohm, G. Ambrosy, K. Lackner, Electric potential difference during laser welding, J. Plasma Phys. 81 (2015), 905810109.

[23] A.D. Brent, V.R. Voller, K.J. Reid, Enthalpy-porosity technique for modelling convection diffusion phase change: application to the melting of pure metal, Numer. Heat Transfer 13 (1998), 297 - 318.

[24] K.C. Mills, Recommended Values of Thermophysical Properties for Selected Commercial Alloys, Woodhead Publishing Ltd, 2002.

[25] B.J. Keene, Review of data for the surface tension of pure metals, Int. Mater. Rev. 38 (1993), 157 - 192.

[26] Y. Li, Y.H. Feng, X.X. Zhang, C.S. Wu, An improved simulation of heat transfer and fluid flow in plasma arc welding with modified heat source model, Int. J. Therm. Sci. 64 (2013), 93 - 104.

[27] R. Rai, J.W. Elmer, T.A. Palmer, T. DebRoy, Heat transfer and fluid flow during keyhole mode laser welding of tantalum, Ti-6Al-4V, 304L stainless steel and vanadium, J. Phys. D Appl. Phys. 40 (2007), 5753. 